\title{
A REVIEW AND BUCKLING ANALYSIS OF STIFFENED PLATE
}

\author{
Ashutosh Kumar ${ }^{1}$, Rachayya R. Arakerimath ${ }^{2}$ \\ ${ }^{I}$ ME Student, Department of Mechanical Engineering, Alard College of Engineering \& Management, Pune, India \\ ashutosh.kr1983@gmail.com \\ ${ }_{2}^{2}$ Professor, Department of Mechanical Engineering, G.H. Raisoni COEM, Pune, India \\ rrarakerimath@gmail.com
}

\begin{abstract}
It happens many times that the structure is safe in normal stress and deflection but fails in buckling. Buckling analysis is one of the method to go for such type of analysis.It predicts various modes of buckling. Plates are used in many applications such as structures, aerospace, automobile etc. Such structures are subjected to heavy uniformly distributed load and concentrated load many times over it's life span. Strength of these structures are increased by adding stiffeners to its plate. This paper deals with the analysis of rectangular stiffened plates which forms the basis of structures. A comparison of stiffened plate and unstiffened plate is done for the same dimensions. In order to continue this analysis various research papers were studied to understand the previous tasks done for stiffened plate. Hyper mesh and Nastran is used in this research work.Buckling analysis is performed for the component with aspect ratio of 2.Rectangular flat bar is used as stiffener
\end{abstract}

Keywords: Stiffened Plate; Dynamic load; Buckling; Aspect ratio;Buckling Analysis.

\section{INTRODUCTION}

In order to increase the strength of structure, plates are one of the common option. These plates are usually two dimensional with thickness very low as compared to other two dimensions. In order to avoid any buckling of these plates, are often strengthened by stiffeners such as angle, bar, etc. Stiffeners mounted on the plate increases the resistance to buckling by increasing the overall stiffness. Such structures are very common in automobile, construction, aerospace etc.

One of the relevant use of plates are in electrically operated overhead cranes. These cranes are having box structure which are rectangular in shape. These box structures consists of two web plates, top and bottom flanges along with diaphragm and stiffeners. Diaphragm divides the box structure basically web plate in panel while stiffener stiffens the web plate.

\section{NEED OF RESEARCH}

Box structure of electrically operated overhead travelling cranes are subjected to heavy load which gets transmitted through the web plates. These structures are normally safe in normal and shear stresses. In order to avoid the buckling effect industries are either increasing the thickness of the plate or adding the stiffeners.

In such a case some analysis and understanding of the buckling behavior of stiffened and unstiffened flat plate is necessary. Hence this paper is considering with aspect ratioof 2. Analysis will be done for two separate plate -one with stiffener and one without stiffener. This analysis will give the nature of plate buckling and corresponding mode shapes.

\section{LITERATURE REVIEW}

\section{A. Review of Papers}

J Rhodes [1], presented a paper on the buckling and post buckling behavior of thin plates. It has made a somewhat concise study with behavior of structure when subjected to local buckling. The author has made a detailed study of all the process being followed till now to understand the plate behavior till now. Still it is clear that there are much more to learn about it.

J Rhodes [2], presented a paper on the buckling of thin plate and early work on rectangular tube. He performed extensive work on the buckling of tubular structure. Post buckling behavior of the tubular thin tube structure was also detailed. Analysis of various sections such as Z,C,and $\mathrm{H}$ sections were done based on previous study. Elastic analysis of plated were performed with simply supported unloaded end and corresponding load deflection curve were outlined. Plastic mechanism analysis were also performed and corresponding constant values were created for Empirical.

N. Raghu Prasad, Jeeoot Singh [3], presented a paper on the buckling of plates with cutout. FEA methods were used to analyze the buckling of these plates. These plates were having simply supported ends. Various method of meshing were used to get optimized result. This analysis was further extended to the study of plate with partial edge compression. These were imposed such that it does not affect the equilibrium loading.. It was concluded in the result that the pattern of stress were varying by small amount for a plate with and without cutout

Ali Reza Pouladkhan,JalilEmadi, Majid Safemehr [4], presented a paper on numerical study of the buckling of thin plates. Equilibrium equation analysis was used to derive the 
buckling load equation. Based on the buckling load equation buckling load was calculated. This exercise was performed for simple support boundary conditions.. The results were further cross verified by using FEA software. Abaqus software was used to do the analysis. Various meshing techniques were used to get the optimized results. Shell elements were used to do the analysis. The numerical solution and FEA solution both were acceptable from matching to each other.

Sang-Rai Cho, Hyun-seung Lee [5], presented a paper for stiffened plate subjected to lateral collision. This paper was basically useful for the ship structure where the plate panels were imposed to lateral loads. Lateral collision experiments were performed on 33 stiffened plates and corresponding results were tabulated. The test model was created with strain gauge located on them. The striker was also prepared with angle welded to it to strike on the localized area. The purpose was to get the pattern of plate buckling because of it. Analytical method was used for further tabulation of kinetic energy and potential energy stored in it.

Ghanialkhenazen, MessaoudSaidani [6], presented a paper on buckling analysis of an isotropic plate which was subjected to in plane patch loading. Total energy concept was being utilized initially which was later analyzed with FEA. Eight nodded quadrilateral element was used for FEA. Based on analysis charts were produced for buckling factor vs aspect ratio. Similar charts were produced for span to width ratio to buckling factor. Another set of loading was used where the loading pattern was point load. Similar graphs were presented as for earlier graph.. The results were in good agreement with previous task performed in the same field.

Khosrow Ghavami, Mohammad Reza Khedmati [7], presented a paper on numerical and experimental investigation of stiffened plate under compression loading. This paper presented behavior of plate when imposed with Compressive force. This analysis was performed beyond elastic limit up to complete failure. In order to perform the analysis plates with stiffener in both longitudinal and transversal direction and only in longitudinal direction were considered. Two series of experiments were performed. In the first series of experiment the stiffeners were having rectangular, $\mathrm{L}$ and $\mathrm{T}$ profiles along with their spacing were studied while in second series the stiffener profiles were of rectangular shapes were considered. In order to perform the analysis FEA software was used as Ansys. In order to get the results testing rigs were also used. Various collapse modes were observed.

Richard Villavicencio, Sang-Rai Cho, Carlos Guedessoares [8], presented a paper on deformation process of web of a girder used in hull structure of small tanker when subjected to lateral impact. Scaled model was used for the analysis purpose. The model was having top plate stiffened by stiffeners. The crushing of model was found to be defined by the deformation of stringers attached to the flanges of model. Impact test were done on the model with the load drop setups. Plastic range on the model sheet was experienced by performing two impact velocities of load on it. Results were tabulated out and were further verified by FEA software LS Dyna. The results were in good agreement to each other.

\section{B. Outcome of Review papers}

Many experiments were performed to know the Buckling behavior of plates with and without stiffeners. Optimal placement of stiffeners were analyzed to understand its effect. Analytical, Numerical and Experimental methods were used whose results were in good agreements to each other.

Various differential equations were produced for different closed and open sections. Main experiments were performed to determine the effect of transverse force on the panel.

\section{BUCKLING ANALYSIS OF PLATE}

\section{Basic Theory.}

Equilibrium method is one of the most common method to do the analysis of plate structure.

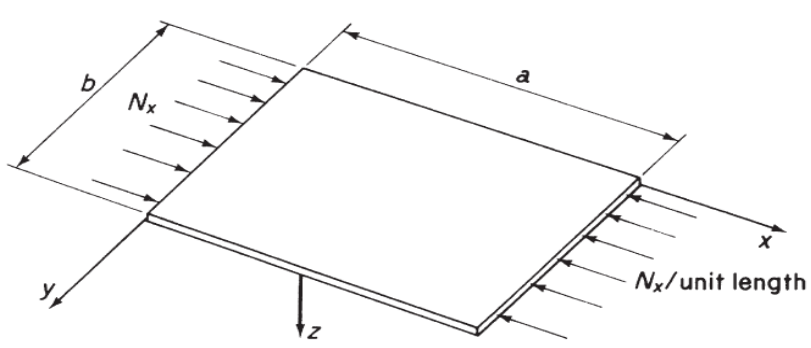

Figure-1 Plate with compressive force along $\mathrm{X}$-axis [9]

For the above shown figure the stadard differential equation comes to be :

$$
\begin{aligned}
& D\left(\frac{d^{4}(\omega)}{d x^{4}}\right)+2 * D\left(\frac{d^{4}(\omega)}{d(x)^{2} d(y)^{2}}\right)+D\left(\frac{d^{4}(\omega)}{d y^{4}}\right) \\
& =N x\left(\frac{d^{2}(\omega)}{d x^{2}}\right)
\end{aligned}
$$

Where,

Nx-Distributed load per unit length on neutral plane.

D-Stiffness of the plate.

$\omega$-Transverse deflection along the thickness of plate

In order to have a trivial solution for such problem following consideration will be there:-

(i) Initial equilibrium condition

(ii) Resultant values of Stress.

(iii)Applied external forces.

Value of these forces can be determined, which will result in non-trivial solution. Minimum of the determined forces will be considered as critical force which will cause the buckling. Further generalizing it will convert problem into an eigenvalue problem. In order to continue further a parameter is multiplied to the stress value $(\mathrm{Nx})$. This parameter is represented as $\lambda$, i.e. 


\section{$\mathrm{Nx}=\lambda \mathrm{Nx}$}

(2)

By substituting equation-2 in equation -1 we get:

$$
\nabla^{4}(w)+\lambda \frac{N x}{D}\left(\frac{d^{2}(w)}{d x^{2}}\right)
$$

Following assumptions will be applicable throughout the discussion:

(i) In initial conditions before load application plate shall be considered undeformed.

(ii) Only external forced will be considered and on the neutral plane.

(ii) Elasticity theory shall describe the stresses with in elastic limit.

(iii) Kirchhoff's theory of plates will be used to form all relations.

Following two cases will be considered for buckling study with boundary conditions as simple supported condition:

(i) Plate without stiffener

(ii) Plate with stiffener.

\section{(i) Plate without stiffener}

Simply supported plate with load acting along $\mathrm{X}$ axis a shown below:

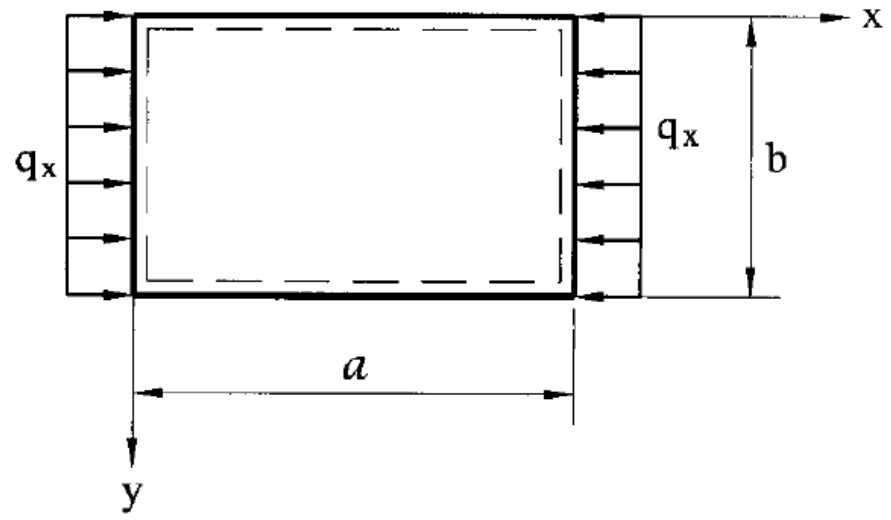

Figure-2 simply supported plate with compressive force along $\mathrm{x}$ axis [9]

Critical load for this case is given as[4]:

$$
\begin{aligned}
q_{x c r}=\frac{4 D \pi^{2}}{b^{2}}, \quad & \sigma_{x, c r}=\frac{q_{x, c r}}{h}, \lambda \\
& =\frac{q_{x c r}}{q_{x}}
\end{aligned}
$$

Where,

$$
D=\frac{E h^{3}}{12\left(1-v^{2}\right)}
$$

$\mathrm{h}=$ Plate thickness

\section{(ii) Plate with stiffener}

Figure-3 shows the required data as per this topic.Uniformly distributed load is applied on the edges as shown below. Longitudinalstiffener may be of any shape

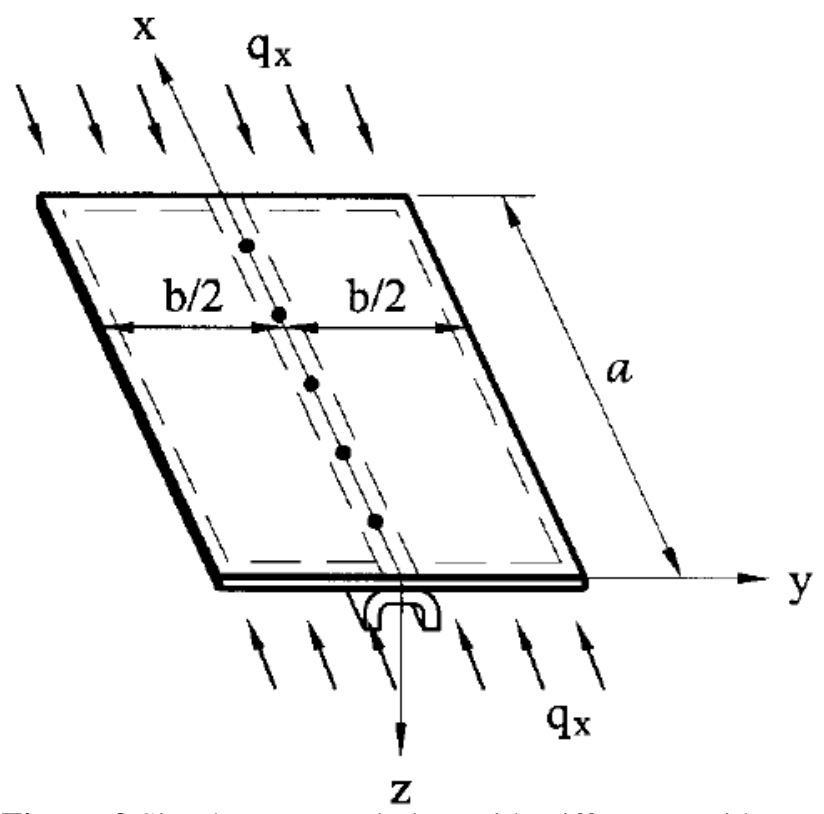

Figure-3 Simply supported plate with stiffener at mid [9]

Stiffener is dividing the plate into to equal parts. We will consider each part with the stiffener.

In order to get the desired solution we will apply the equation 2 to the one half of the plate with the standard solution as below

$w(x, y)$

$=F_{Y} \sin \left(\frac{m \pi x}{a}\right)$

Putting the above equation in equation-2 we get:

$D\left(\frac{d^{4}\left(F_{Y}\right)}{d y^{4}}\right)-2\left(\frac{m \pi}{a}\right)^{2} \frac{d^{2} F(y)}{d y^{2}}+\left(\frac{m \pi}{a}\right)^{2}\left[\left(\frac{m \pi}{a}\right)^{2}-\frac{q_{x}}{D}\right.$

$=0$

The solution comes in the form

$$
\begin{gathered}
F(y)=A 0 \cosh \alpha y+A 1 \sinh \alpha y+A 2 \cos \beta y \\
+A 3 \sin \beta y \quad(7)
\end{gathered}
$$

Where,

$$
\begin{gathered}
\alpha=\left[\mu\left(\mu+\left(\frac{q_{x}}{D}\right)^{0.5}\right]^{\frac{1}{2}}, \beta=\left[\mu\left(\left(\frac{q_{x}}{D}\right)^{0.5}-\mu\right]^{\frac{1}{2}} \& \mu\right.\right. \\
=\frac{m \pi}{a} \text { (8) }
\end{gathered}
$$

At $\mathrm{y}=0.5 \mathrm{~b}$ we get :

$\omega=0, \frac{d^{2} w}{d y^{2}}=0$ at $y$

$$
=0.5
$$

Since we have considered half part of plate with stiffener for analysis the plate and stiffener shall be assumed to bend together and finally get buckled. The pattern must possess symmetry about $\mathrm{X}$ axis. This results in the below mentioned condition:

$\frac{d w}{d y}=0$, at $y$

$=0$

The difference in the reaction forces from the two strip of the plate will be represented as below:

$R_{y}=-D\left[\frac{d^{3} w}{d y^{3}}+(2-v) \frac{d^{3} w}{d y d x^{2}}\right]$ 
This reaction will get transferred to the stiffener. Since all the component are of same material, first part of above equation will be of importance only while the second part will get cancelled.

The governing equation for plate with compressive force is given as below:

$E I_{i} \frac{d^{4} w}{d x^{4}}+\sigma_{x} A_{i} \frac{d^{2} w}{d x^{2}}+2 D \frac{d^{3} w}{d y^{3}}=0$, at $y$

$$
=0 \quad \text { (12) }
$$

Where Ii is moment of inertia while Ai is the area of the stiffener.

Few parameters are required to be introduced here as below:

$$
\begin{aligned}
& \gamma=\frac{E I_{i}}{D b}, \zeta=\frac{a}{b}, \delta \\
& =\frac{A_{i}}{b h}
\end{aligned}
$$

Introducing the equation -7 into the conditions of equation9, $10 \& 13$, we get a set of homogeneous equations for A0, A1, $\mathrm{A} 2$, A3. For solving these equations we will equate the determinant to zero, which will result in the below equation:

$$
\begin{aligned}
\left(\frac{1}{b \alpha} \tanh \left(\frac{b \alpha}{2}\right)-\right. & \left.\frac{1}{b \beta} \tanh \left(\frac{b \beta}{2}\right)\right)\left(\frac{\gamma m^{2}}{\alpha^{2}}-K \delta\right) \frac{\left(m^{2} \pi^{2}\right)}{\alpha^{2}} \\
& -\frac{4 m \sqrt{k}}{\alpha} \\
& =0
\end{aligned}
$$

Where,

$$
k=\frac{\sigma_{x, c r}}{\sigma_{x, E}}
$$

And,

$\sigma_{x, c r}=\frac{q_{x, c r}}{h}, \sigma_{x, E}=\frac{4 D \pi^{2}}{b^{2} h}(15)$

For $m=1 \& \zeta>2$, its solution using the equation 13 the expression for critical stress is achieved as :

$\sigma_{x, c r}=\frac{\pi^{2} D}{b^{2} h} \frac{\left(1+\zeta^{2}\right)^{2}+2 \gamma}{\zeta^{2}(1+2 \delta)}(16)$

\section{Analytical analysis.}

In order to perform the analytical analysis we will consider the two pre discuss cases to understand the stiffener effect on buckling view)

.Input Data (Kindly refer Figure2 and 3 for pictorial

Table-1 Input data sheet

\begin{tabular}{|c|c|c|}
\hline Parameter & Case- 1 & Case-2 \\
\hline $\mathrm{a}(\mathrm{m})$ & 2400 & 2400 \\
\hline $\mathrm{b}(\mathrm{m})$ & 1200 & 1200 \\
\hline $\mathrm{h}(\mathrm{m})$ & 0.006 & 0.006 \\
\hline stiffener $(\mathrm{mm})$ & $\mathrm{NA}$ & $50 \mathrm{X} 5-2400$ \\
\hline $\mathrm{E}(\mathrm{Mpa})$ & $2.1-\mathrm{e} 5$ & $2.1-\mathrm{e} 5$ \\
\hline Poisson's ratio & 0.3 & 0.3 \\
\hline Load on the edge b(N) & 500 & 500 \\
\hline
\end{tabular}

Output Data (Result)

Table-2 Output data sheet

\begin{tabular}{|c|l|l|l|}
\hline & Case-1 & Case-2 & $\begin{array}{c}\text { Resource } \\
\text { equation }\end{array}$ \\
\hline $\mathrm{q}_{\mathrm{xcr}}(\mathrm{N} / \mathrm{mm})$ & 114 & 244 & \\
\cline { 1 - 2 } Eigen Value & 5.2 & 11.2 & $\begin{array}{c}\text { Equation-4 \& } \\
\text { Equation-15 }\end{array}$ \\
\hline
\end{tabular}

\section{E. FEA Analysis of Case-1 and Case-2}

In FEA Buckling analysis is done and the result is termed in term of Eigen value. Buckling analysis of plate, Beams or column is divided into two types:

(i) Linear Buckling

(ii) Non-Linear Buckling

This paper deals with linear buckling. It estimates the maximum loads that can be supported prior to the structural instability or collapse. This is also known as Eigen value buckling. The characteristic equation of buckling analysis is given by below equation:

$[K] \emptyset_{i}$

$=\lambda[M] \emptyset_{i}$

Where,

$\mathrm{K}=$ stiffness matrix; $\lambda=$ Eigen Value; $\mathrm{M}=$ Mass matrix

$\emptyset_{i}=$ Eigen value or mode shapes

Initially linear static analysis is required to be performed. It then formulate $\mathrm{M}$ matrix from the reference load and then the below mentioned expression is formed:

$[k-\lambda M] \phi$

$=0$

Then Block Lancoz method is used to solve the lowest Eigen value to accomplish the analysis. Critical load is given AS below:

$$
\begin{aligned}
& P c r=\lambda_{c r} P ; \lambda_{c r} \\
& =\frac{P c r}{p}
\end{aligned}
$$

Where,

Pcr $=$ Critical load, $\mathrm{P}=$ Applied load, $\lambda_{c r}=$ Critical Eigen value

If Critical Eigen value is less than 1 then there will be buckling else no buckling. The displacement calculated in Eigen value will predict the buckling mode.

Hypermesh and Nastran software were used to do the analysis. 4 node shell elements were used to discretize the complete plate structure. After applying the boundary condition Nastran was used to do the post processing and the results of first 6 modes were divided into twocases.

- Case-1-Plate without stiffener

- Case-2.-Plate with stiffener.

Element type used is 4 node shell element. PShell is the element property used to give the thickness value. 


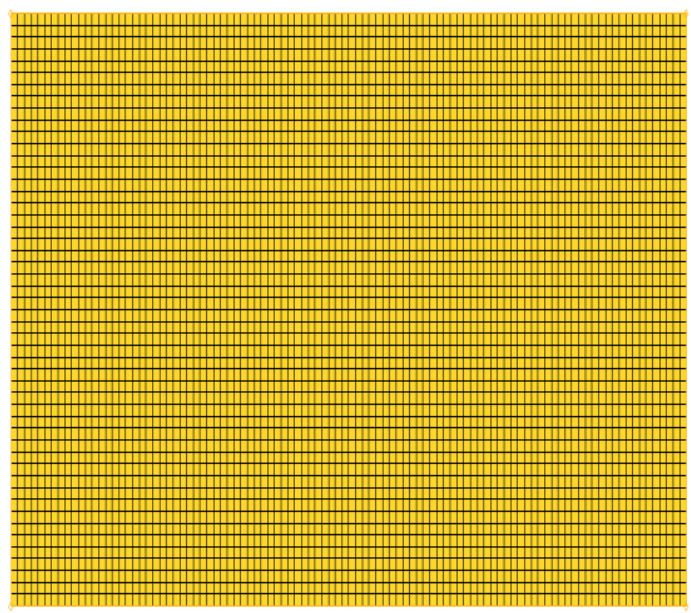

Figure-4 Mesh model of case-1

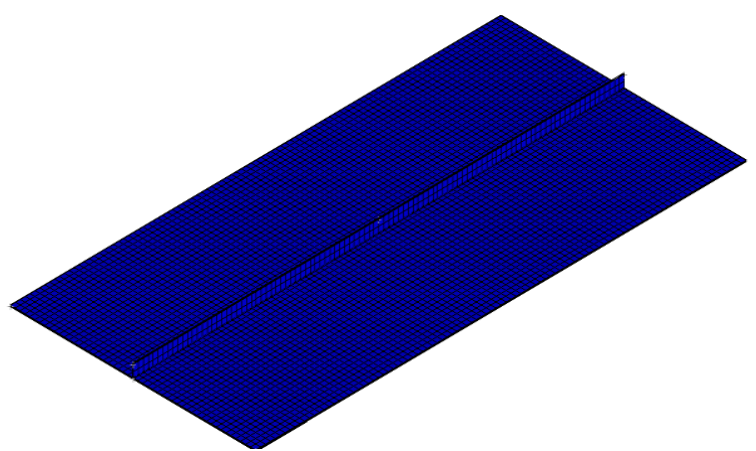

Figure-5 Mesh model of Case-2

- Eigen Value result of FEA analysis

- First Eigen Value

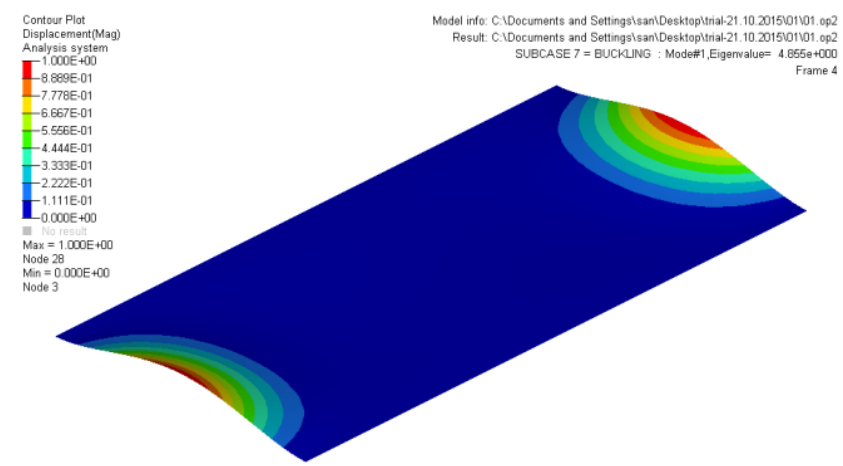

Figure-6 Plate without Stiffener

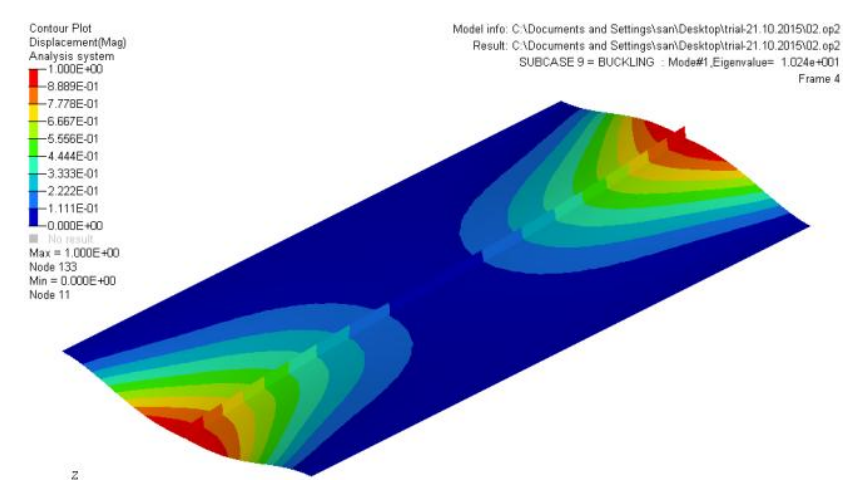

Figure-7 Plate with Stiffener

\section{- Second Eigen Value}

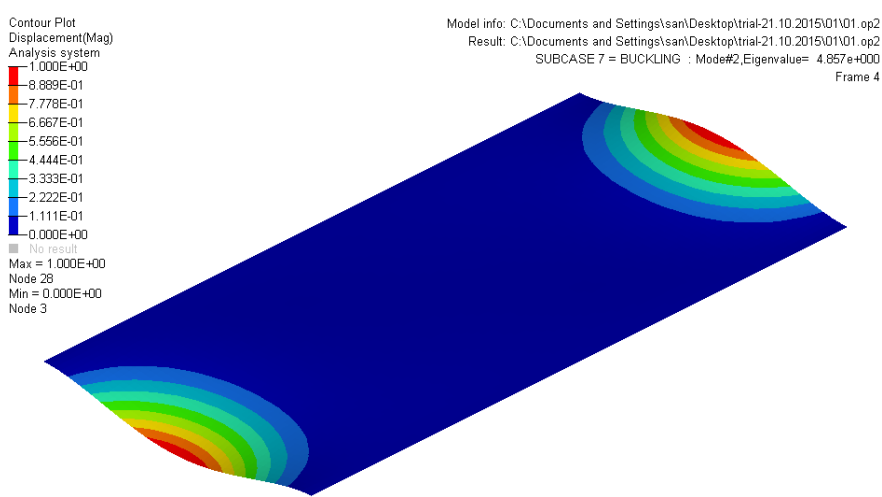

Figure-8 Plate without Stiffener

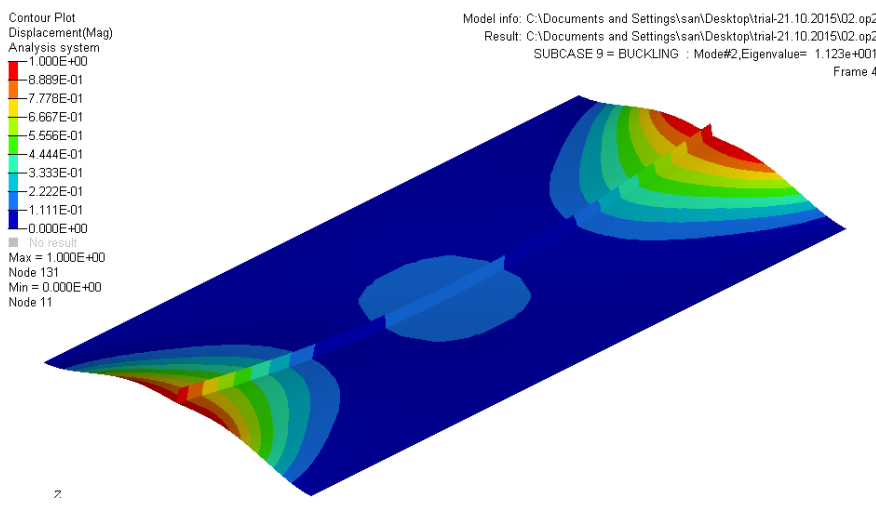

Figure-9 Plate with Stiffener

\section{- Third Eigen Value}

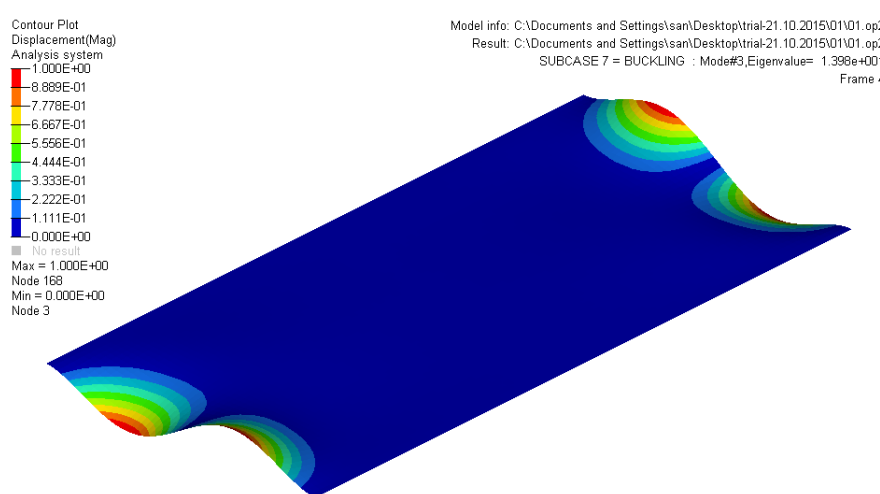

Figure-10 Plate without Stiffener

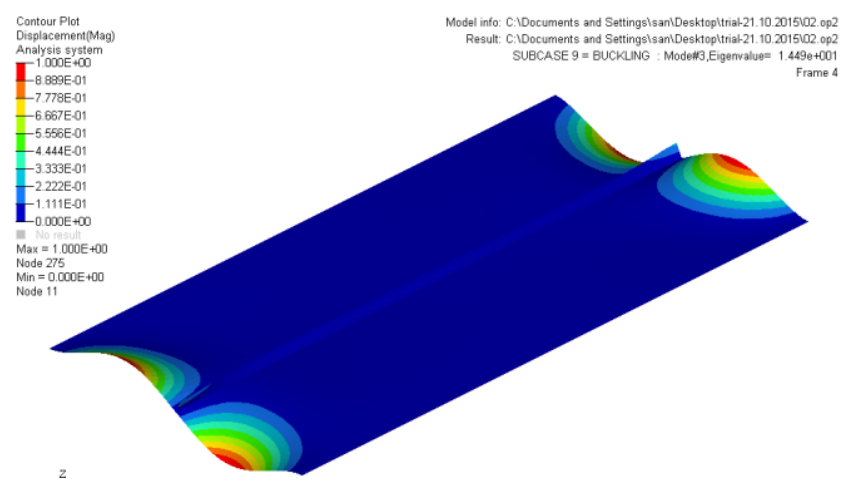

Figure-11 Plate with Stiffener 


\section{- Fourth Eigen Value}

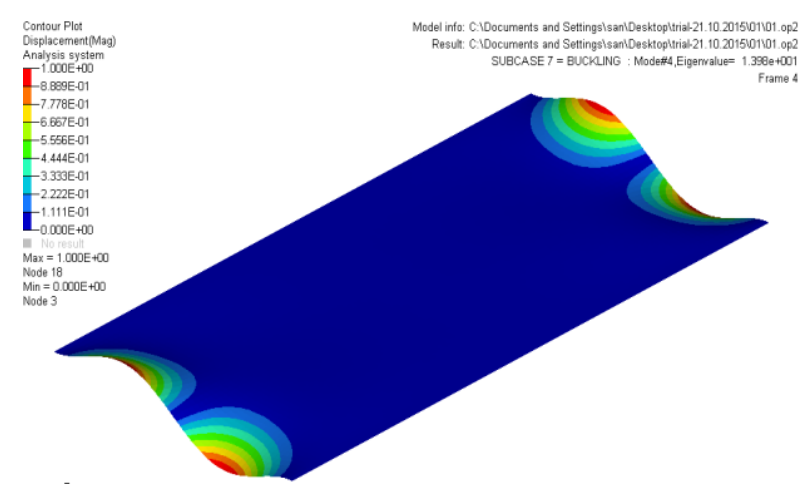

Figure-12 Plate without Stiffener

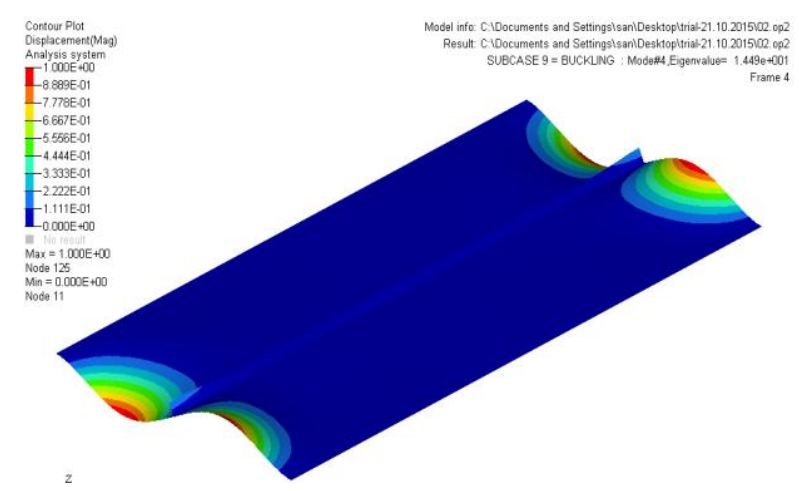

Figure-12 Plate with Stiffener

\section{- Fifth Eigen Value}

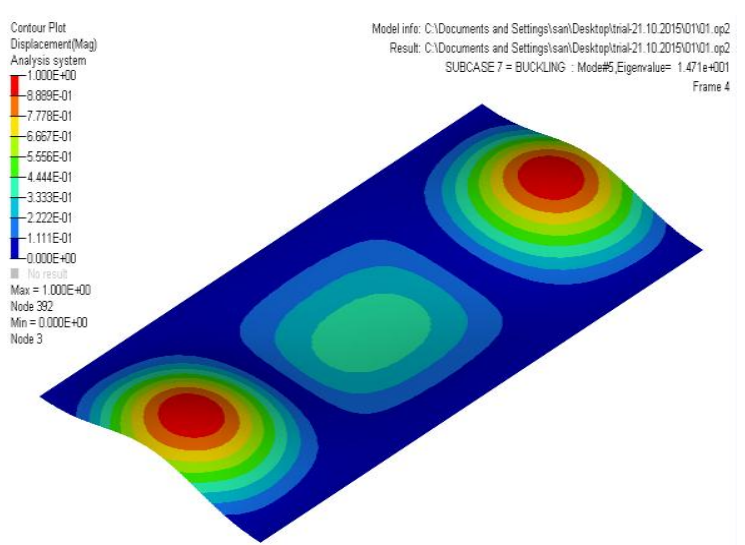

Figure-13 Plate without Stiffener

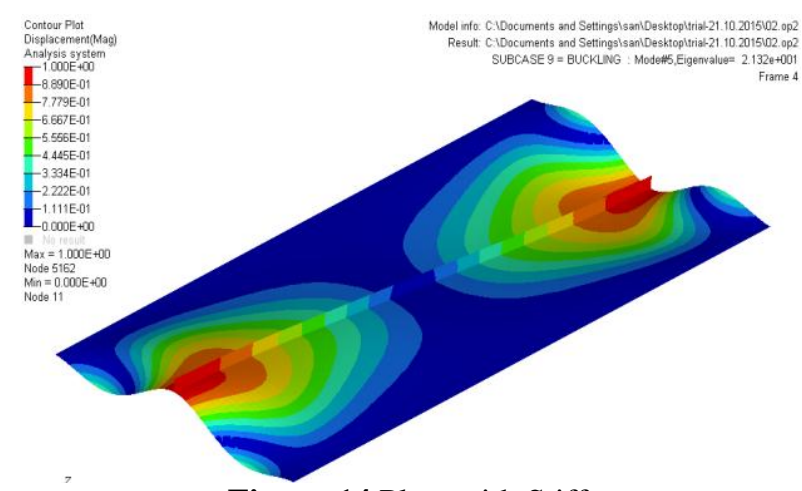

Figure-14 Plate with Stiffener

\section{- Sixth Eigen Value}

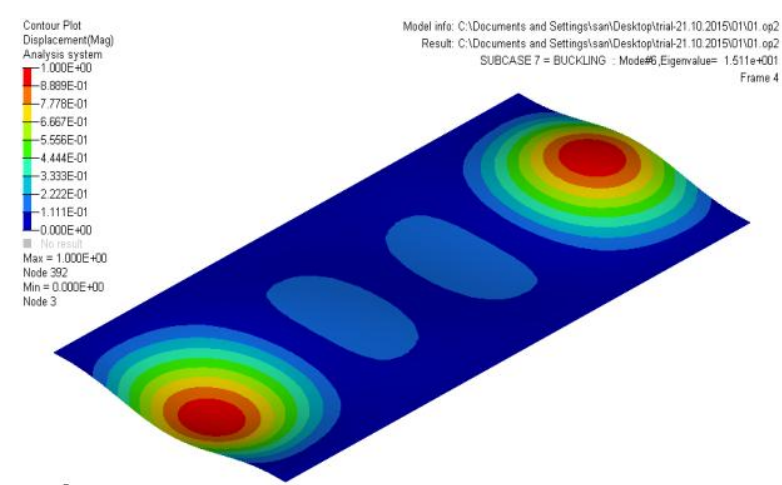

Figure-15 Plate without Stiffener

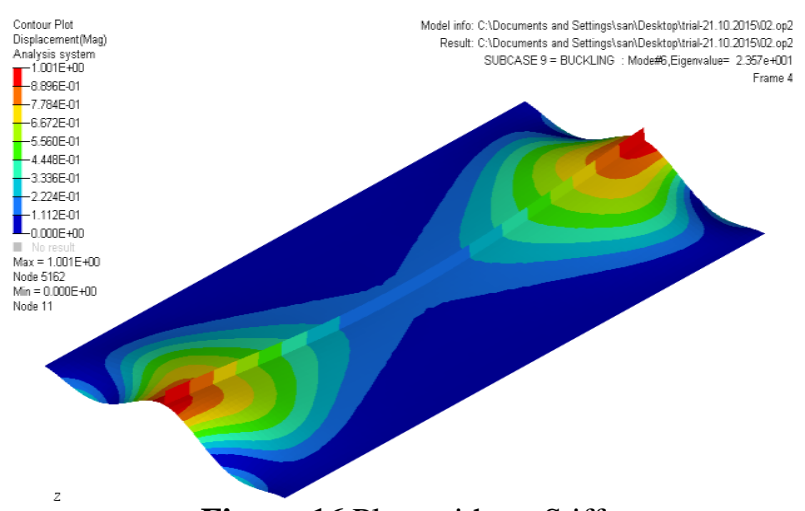

Figure-16 Plate without Stiffener

\section{RESULTS AND COMPARISON}

FEA Results Plotting

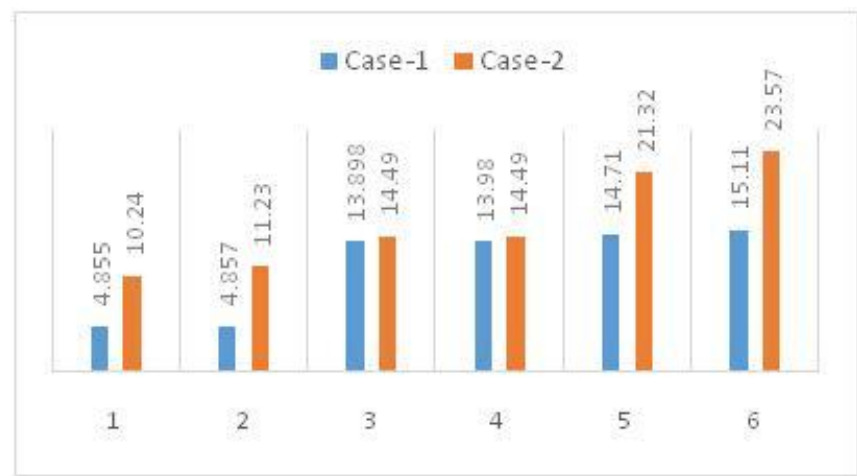

Table-3 Eigen value comparison sheet for Case-1 \& Case-2

The difference between the analytical calculation of Eigen value and FEA result for the first Eigen value is 7\%. It can be further reduced by refining the mesh size further.

\section{CONCLUSION}

Analytical calculation and FEA results were in good agreement to each other. It was found that Eigen value is increasing with the addition of stiffeners.

Buckling analysis shows almost similar pattern for both cases with different mode shapes.Eigen value values are increasing according to the addition of number of stiffener is of prime importance as the other modes are having very safe value. 
There is further analysis scope to analyze the same case with different stiffeners geometry.

\section{ACKNOWLEDGMENT}

I wish to express my sincere thanks to Mechanical engineering department of ACEM, Dr. S.B. Padwal, Principal (ACEM, Pune) and Prof. V.R. Bajaj, HOD (Mechanical department) for their help and faith toward my research. They provided all facilities to do this task.

\section{REFERENCES}

[1] J Rhodes, Some observation of the post-buckling behavior of thin plates and thin walled members, Science direct, 2003

[2]. J Rhodes, Buckling of thin plate members-and early work on rectangular tubes, Elsevier science Ltd,2002.

[3]. N. Raghu Prasad, Jeeoot Singh, Buckling analysis of Rectangular plates with cutout and partial edge compression,IARJSET,2015.

[4]. Ali Reza Pouladkhan, JalilEmadi, Majid Safemehr, Numerical study of buckling of thin plates, World academy of science, engineering and technology,2011.

[5]. Sang-Rai Cho, Hyun-seung Lee, Exprimental and analytical investigations on the response of stiffened plates subjected to lateral collision, Sciencedirect,2009.

[6]. Ghanialkhenazen, MessaoudSaidani, Finite element analysis of linear plates buckling under in-plane patch loading, Researchgate, 2010.

[7]. Khosrow Ghavami, Mohammad Reza Khedmati, Numerical and Experimental investigations on the compression behavior of stiffened plates,ScienceDirect, 2006

[8]. Richard Villavicencio, Sang-Rai Cho, Carlos Guedessoares, Deformation process of web girders in smallscale tanker double hull structures subjected to lateral impact, ResearchGate,2013.

[8].A.K.L.Shrivastava， P.K.Datta,A.H.Seikh， Transverse vibration of stiffened plates with cutouts subjected to inplane uniform edge loading at the plate boundary, IOS Press, 2002.

[9].EsuardVentsel, Theodor Krauthammer, Thin Plates and shell, 2001 